EPJ Web of Conferences 41, 05003 (2013)

DOI: $10.1051 /$ epjconf/20134105003

(C) Owned by the authors, published by EDP Sciences, 2013

\title{
Exploring the polarization degrees of freedom in collinear two-dimensional infrared spectroscopy
}

\author{
J. Réhault ${ }^{1}$ and J. Helbing ${ }^{1}$ \\ ${ }^{1}$ University of Zurich, Physical Chemistry Institute, CH-8057 Zurich, Switzerland
}

\begin{abstract}
We exploit the polarization degrees of freedom in order to optimize interferometer-based 2D-IR spectroscopy in the pump-probe geometry. Signals can be amplified, scattering background can be fully eliminated and intramolecular angles can be determined with improved accuracy. Simple manipulation of the polarization of all four fields gives direct access to individual tensor elements and dispersive signals.
\end{abstract}

\section{Introduction}

Fourier transform (FT) two dimensional infrared (2D-IR) spectroscopy in the collinear geometry is a simple and reliable technique that leads directly to purely absorptive spectra and requires aligning only two beams at the sample [1]. In a simple implementation we introduced in our laboratory, the pair of pump pulses is generated by a fast-scanning interferometer with automatic determination of their relative phase for easy retrieval of the 2D-IR spectra [2]. In the standard configuration the signals in the pump-probe configuration are inherently weaker than in a photon echo geometry, and one can access only the two polarisation tensors $\langle\mathrm{XXYY}>$ and $\langle\mathrm{YYYY}\rangle$, corresponding to perpendicular and parallel orientation of pump pulses and the probe and local oscillator pulse. However, it has been shown that one can amplify the 2D-IR signals by placing a polarizer in the probe beam behind the sample $[3,4]$. In this contribution, we manipulate the molecular response by controlling independently the polarisation of the two pump pulses as well as the probe- and the heterodyning fields. We show how to interpret polarization-enhanced 2D-IR signals to obtain structure information, eliminate selected peaks in the spectra, suppress scattering and measure dispersive spectra directly.

\section{Methods and Results}

In 2D-IR spectroscopy a signal field is generated by three incident fields, two coming from the pump beams and one from the probe beam $\left(\mathrm{E}_{\text {signal }} \alpha \mathrm{E}_{\text {pump }}^{2} \cdot \mathrm{E}_{\text {probe }}\right)$. With collinear pump-pulses, this signal is self heterodyned by the probe beam, producing intensity changes on the detector proportional to $\mathrm{E}_{\text {signal }} \cdot \mathrm{E}_{\text {probe }}$. Thus, the third order signals in the pump-probe geometry are limited by the probe intensity, which is limited by the dynamic range of the detector. Attenuating the probe usually attenuates the signal at the same time. One way of circumventing these limitations is to produce a signal field that is polarized perpendicular to the probe field. It is then possible to place the sample between nearly crossed polarizers, allowing to work with an intense probe beam at the sample and to control its intensity on the detector (see Fig. 1) without significant signal attenuation [3].

This is an Open Access article distributed under the terms of the Creative Commons Attribution License 2.0, which permits unrestricted use, distribution, and reproduction in any medium, provided the original work is properly cited. 

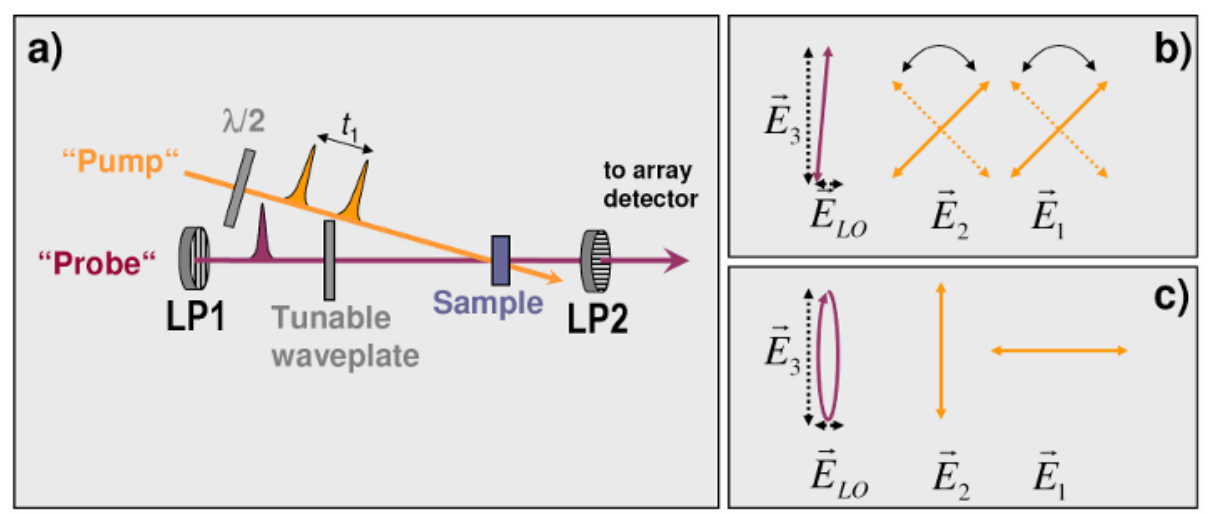

Fig. 1. a) Schematic representation of the setup. b) Orientation of the plane of polarization of the laser pulses for signal amplification and angle measurements. The probe beam polarisation is tilted by a small angle $\beta$, and only a small fraction leaks through LP2 (horizontal). The polarisation of the pump pulse pair may be flipped with a half wave plate for angle determination and scattering suppression. c) Beam polarizations for dispersive measurements: The local oscillator is now phase shifted by $\pi / 2$ with respect to the third field.

When the angle between the quasi-crossed polarizers LP1 and LP2 is $\pi / 2+\beta$ and the polarization of both pump pulses is set to $\pi / 4$ with respect to LP2 as shown in Fig. 1b, the 2D-IR signal can be written in analogy to transient absorption spectroscopy $[4,5]$ :

$$
\Delta A_{\beta}=\left(\Delta A_{/ /}+\Delta A_{\perp}\right)+\left(\Delta A_{/ /}-\Delta A_{\perp}\right) \cdot \operatorname{Cot}(\beta)=\Delta \bar{A}+L D \cdot \operatorname{Cot}(\beta)
$$

where $\Delta A_{\|}$and $\Delta A_{\perp}$ correspond the conventional 2D-IR signals for parallel and perpendicular probing respectively. We see from eq. 1 that it is the linear dichroism component, defined as the difference between signals obtained for parallel and perpendicular probing that is amplified in the crossed polarizer scheme. As the angle $\beta$ is changed, different peaks in the spectrum scale differently, depending on their anisotropy. This makes it possible to selectively amplify, eliminate or change the sign of peaks, as illustrated in Fig. 2. However, in order to properly interpret these signals, at least two different measurements should be made.
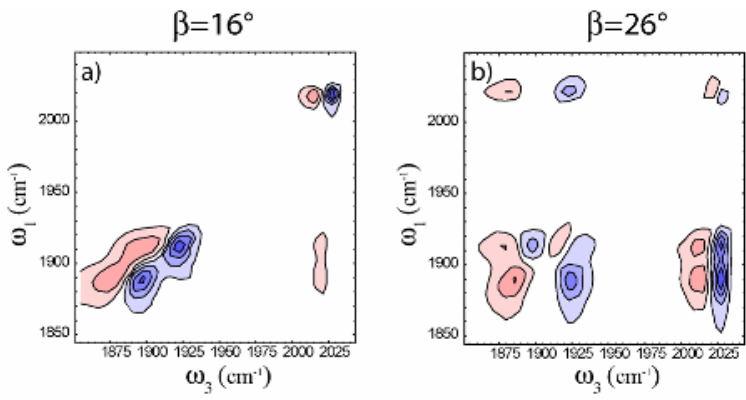

Fig. 2. 2D-IR spectra of the Rhenium complex $\operatorname{Re}(b p y)(C O)_{3} B r$, with population time $t_{2}=2 p s$. a) Elimination of the cross peaks b) Elimination of the diagonal peaks.

Reversing the sign of $\beta$, or, equivalently, switching the pump polarization between $\pi / 4$ and $-\pi / 4$ changes the sign of the LD component of the signal. Subtraction of these two spectra $\Delta A_{+}$and $\Delta A_{-}$ eliminates the isotropic component and can be used to remove background from solvents, or other isotropic signals. Either the sign of the heterodyning field or that of the (LD) signal can be reversed. The latter possibility is particularly interesting, as it can be used to completely remove any scatter contribution from the 2D-IR spectrum with only two measurements [5]. In analogy to anisotropy 
experiments, the two measurements can also be combined to define the metric $\alpha^{\prime}=$ $\tan \beta\left(\Delta A_{+}-\Delta A_{-}\right) /\left(\Delta A_{+}+\Delta A_{-}\right)$. This makes it possible to retrieve the angle $\phi$ between excited and probed transition dipole moments from strongly amplified $2 \mathrm{D}$ signals via the relation

$$
\cos ^{2} \phi=\frac{3 \alpha^{\prime}+1}{3-\alpha^{\prime}}
$$

We can add another degree of freedom by introducing polarizers instead of beamsplitters in the interferometer, which is then generating two perpendicularly polarized pump pulses. This way we can measure individual tensor elements $\langle\mathrm{XYXY}>$ or $\langle\mathrm{YXXY}\rangle$ that are usually not accessible independently in a pump-probe set-up. In addition, by crossing to extinction the polarizers in the probe beam, and introducing a tunable wave-plate at $45^{\circ}$, we have produced a heterodyning field that is phase shifted by $\pi / 2$ relative to the probe beam at the sample (see Fig. 1c). In this way we could measure directly the dispersive part of the molecular response, as shown for the asymmetric stretch vibration of azide in water in Fig. $3 \mathrm{~b}$.
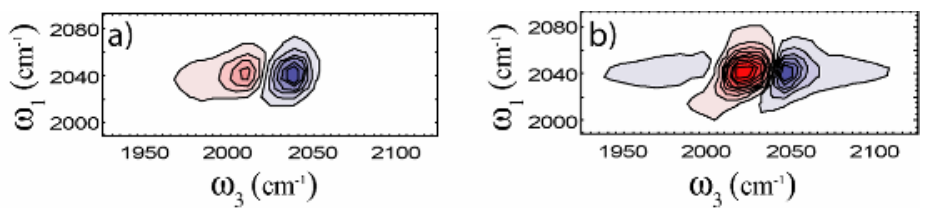

Fig. 3. a) absorptive and b) dispersive $2 \mathrm{D}-\mathrm{IR}$ spectra of the asymmetric stretch of $\mathrm{N}_{3}{ }^{-}$in water. In each case, the isolated $<\mathrm{XYXY}>$ tensor was measured using crossed-polarized pump beams.

In both Fig. $3 \mathrm{a}$ and $3 \mathrm{~b}$ we plot the cosine-Fourier transform of the raw data $I^{a b s}\left(\mathrm{t}_{1}, \omega_{3}\right)$ and $I^{d i s p}\left(\mathrm{t}_{1}, \omega_{3}\right)$, respectively, which we denote by $\Delta A_{\mathrm{Cos}}\left(\omega_{1}, \omega_{3}\right)$ and $\Delta D_{\mathrm{Cos}}\left(\omega_{1}, \omega_{3}\right)$. The sine-transform of $I^{\text {disp }}\left(\mathrm{t}_{1}, \omega_{3}\right)$ yields a third signal $\Delta D_{\operatorname{Sin}}\left(\omega_{1}, \omega_{3}\right)$ which can be used to construct the rephasing $(\mathrm{R})$ and non-rephasing (NR) 2D-IR spectra:

$$
\begin{aligned}
& \mathrm{R}\left(\omega_{1}, \omega_{3}\right)=\Delta A_{\operatorname{Cos}}\left(\omega_{1}, \omega_{3}\right)+\Delta D_{\operatorname{Sin}}\left(\omega_{1}, \omega_{3}\right) \\
& \mathrm{NR}\left(\omega_{1}, \omega_{3}\right)=\Delta A_{\operatorname{Cos}}\left(\omega_{1}, \omega_{3}\right)-\Delta D_{\operatorname{Sin}}\left(\omega_{1}, \omega_{3}\right) .
\end{aligned}
$$

Note that only directly measured data are needed to obtain these signals. If only the absorptive data is recorded, on the other hand, two pseudo Kramers-Kronig transforms along $t_{3}$ and $t_{1}$ are necessary to compute $\Delta D_{\operatorname{Sin}}[4]$.

\section{Conclusions}

We have presented a set of modifications of a pump-probe 2D-IR scheme, exploiting fully the polarisation degrees of freedom. They allow us to approach the sensitivity and versatility of a photon echo set-up, are easy to implement and can be generalized to higher order experiments, for example transient 2D-IR or 3D-IR spectroscopy.

\section{References}

1. L. P. DeFlores, R. A. Nicodemus, and A. Tokmakoff, Opt. Lett. 32, 2966 (2007).

2. J. Helbing and P. Hamm, J. Opt. Soc. Am. B 28, 171 (2011).

3. W. Xiong and M. T. Zanni, Opt. Lett. 33, 1371 (2008).

4. J. A. Myers, K. L. Lewis, P. F. Tekavec, and J. P. Ogilvie, Opt. Express 16, 17420 (2008).

5. J. Réhault and J. Helbing, J. Chem. Phys. 134, 124516 (2011)

6. J. Réhault and J. Helbing, (2012) submitted. 